\title{
Prestack Inverse-Ray Imaging of A 2D Homogeneous Layer: A Tutorial Study
}

\author{
Tan K. Wang ${ }^{1, *}$ \\ (Manuscript received 7 May 2002, in final form 26 September 2002)
}

\begin{abstract}
Traveling directions of an inverse ray for the common shot, the common midpoint and the common offset gathers are explicitly determined from the geometry of reflected rays and an envelope of two reflected ellipses in a 2D homogeneous irregular layer. I find that a common shot gather and a common offset gather can be applied to image the structural velocity and interface. However, due to the symmetry of the travel-time hyperbola, a common midpoint gather is not suitable for structural imaging. Furthermore, from a common offset gather, the poststack inverse ray is proved as a special case of the prestack inverse ray.

Error analysis of the prestack inverse ray indicates that the method of elliptic envelope provides more accurate imaging at far offsets than the method of ray geometry if the travel-time picks are limited along a reflected hyperbola. Alternatively, when the travel-time picks are sufficient, the method of ray geometry is superior to the method of elliptic envelope. The prestack inverse ray is also applied to image a sedimentary basin. The results suggest that the best way for applying the prestack inverse ray is to determine the layer velocity from ray geometry and to image the structural interfaces by considering both methods.
\end{abstract}

(Key words: Common offset, Common shot, Complex structure, Image source, Prestack depth migration, Reflected ellipse, Travel time)

\section{INTRODUCTION}

Prestack depth migration has been widely applied for the multi-channel seismic (MCS) data processing of the steep dips and faulted structures (Gray et al. 2001). Although the computing speed of the prestack depth migration has been enhanced by the ray-based migration (Operto et al. 2000; Hill 2001), its applications on the migration velocity analysis and the realtime monitoring are still limited (Donihoo et al. 2001). On the other hand, by using the poststack

\footnotetext{
${ }^{1}$ Institute of Applied Geophysics, National Taiwan Ocean University, Keelung, Taiwan, ROC

* Corresponding author address. Prof. Tan K. Wang, Institute of Applied Geophysics, National Taiwan Ocean University, Keelung 20224, Taiwan, ROC; E-mail: tkwang@mail.ntou.edu.tw
} 
data, the geometrical migration of reflected signals or the inverse-ray method (May and Covey 1981) has provided an efficient way to image the crustal interfaces (Warner 1987; Raynaud 1988). The key point of the inverse-ray method is to determine a take-off angle of an inverse ray from the zero-offset travel-times. Recently, inverse rays were applied to enhance the capability of the poststack depth migration (Hua and McMechan 2001). Furthermore, the mathematical derivation and the physical meaning of the zero-offset inverse rays in 2D multilayered structures were proposed (Wang and Tan 2002).

Until now, few studies have been conducted on the inverse ray for the case of the non-zero offset although the reflected signals in the MCS data can be fully used for prestack imaging of complex structures. By considering envelopes of reflected ellipsoids from the common shot gathers, Pivot (1997) first proposed a 3D prestack geometrical migration to image a 3D homogeneous layer. However, the main drawback of his prestack geometrical migration was the inefficiency of computing the reflected ellipsoids. The aims of this paper are to present a tutorial study of the prestack inverse-ray method and to image the complex structures efficiently from the reflected travel-times of the MCS data.

\section{THEORY}

A reflected point at an interface is imaged through an inverse ray if directions of the reflected ray emitting from a source $(S)$ and a receiver $(\mathrm{R})$ can be determined. In the following, the ray geometry and the reflected envelopes of a common shot gather, a common midpoint gather and a common offset gather are investigated for determining the directions of an inverse ray. For simplicity, I present the prestack inverse-ray imaging of a $2 \mathrm{D}$ homogeneous irregular layer with a constant velocity of $V$. The sources and the receivers in a MCS survey are also assumed at a flat surface $(z=0)$.

\subsection{Ray Geometry}

A virtual source $\left(S^{\prime}\right)$ and a virtual receiver $\left(R^{\prime}\right)$, reflected images of a source and a receiver to an interface with a dip of $\alpha$, are applied to elaborate the geomery of the non-zero offset rays (Fig. 1). For determining the direction of an inverse ray, I project the virtual source and the virtual receiver onto the flat surface as denoted by $S$ " and $R$ " in Fig. 1, respectively. Thus, by considering the right triangles $R S$ ' $S$ ' and $S S$ ' $S$ " in three types of the source-receiver gathers, the direction of an inverse ray emitting from the receiver can be written as

$$
\cos (\angle R)=-\frac{\overline{R S}+\overline{S S^{\prime}} \sin \alpha}{\overline{R S^{\prime}}}, \quad \sin (\angle R)=\frac{\overline{S S^{\prime}} \cos \alpha}{\overline{R S^{\prime}}} .
$$

Similarly, by investigating the right triangles $S R R^{\prime}$ " and $R R$ ' $R$ ", the direction of an inverse ray shooting from the source is

$$
\cos (\angle S)=\frac{\overline{S R}-\overline{R R^{\prime}} \sin \alpha}{\overline{S R^{\prime}}}, \quad \sin (\angle S)=\frac{\overline{R R^{\prime}} \cos \alpha}{\overline{S R^{\prime}}} .
$$


(a)

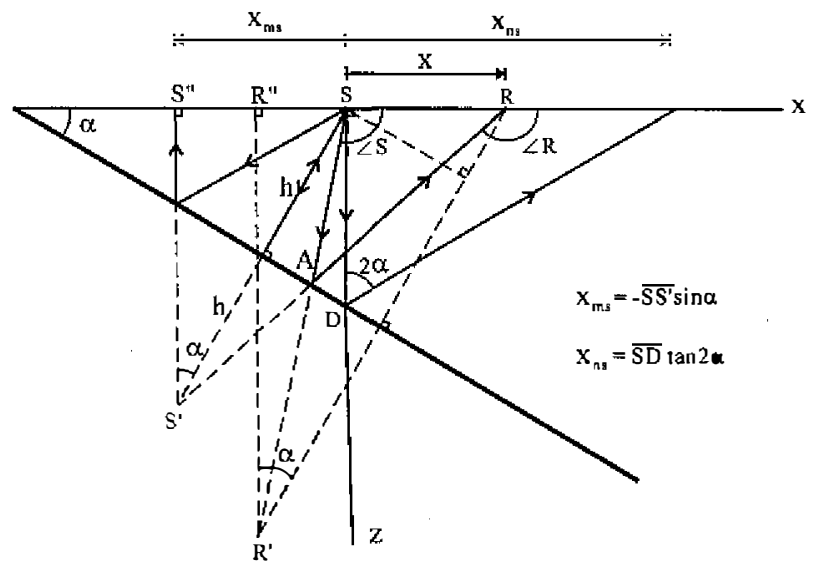

(b)

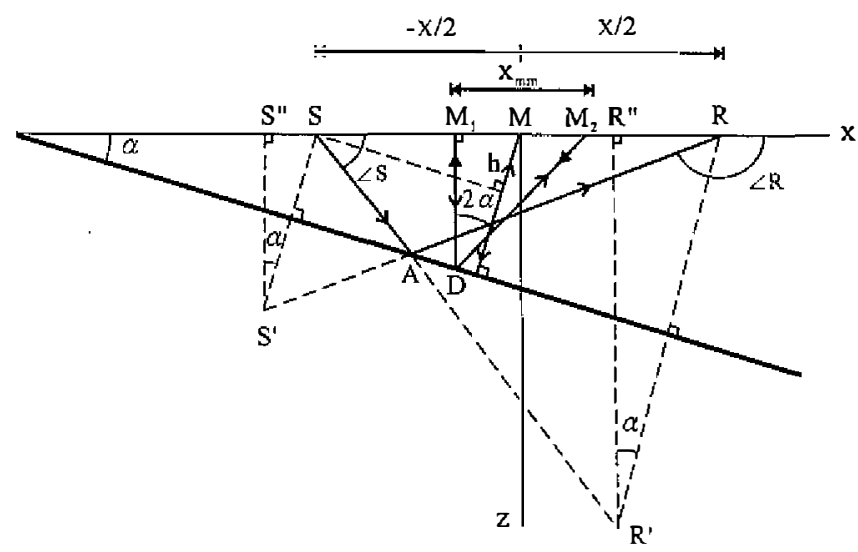

(c)

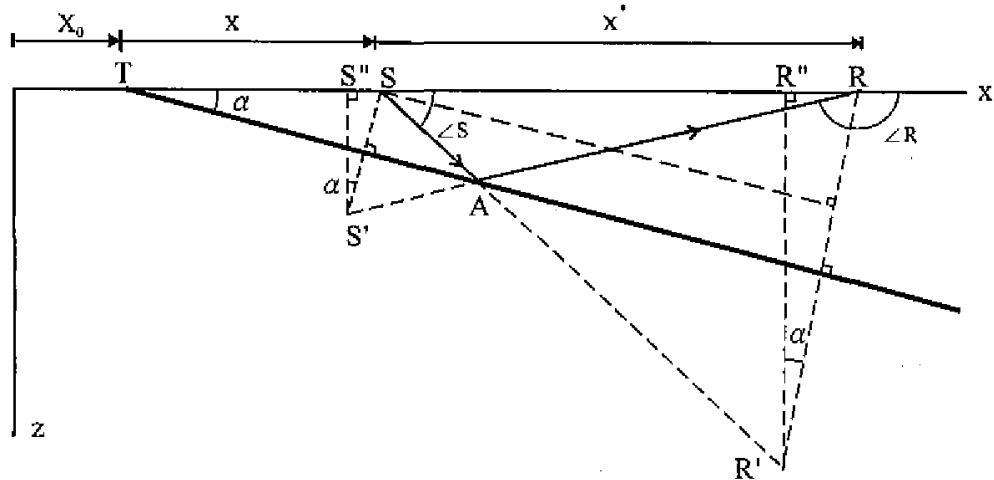

Fig. 1. Reflected rays of (a) a common shot gather, (b) a common midpoint gather, and (c) a common offset gather travel in a homogeneous layer with a dip angle of $\alpha$. A virtual source $\left(S^{\prime}\right)$ and a virtual receiver $\left(R^{\prime}\right)$ are located as the reflected images of a source $(S)$ and a receiver $(R)$ to the dipping interface, respectively. The projection of the virtual source and receiver onto the surface are denoted by S" and R", respectively. 
Therefore, in terms of the ray direction, the reflected point with respect to the source point can be derived,

$$
A_{x}=\frac{\cot (\angle S) \overline{S R}}{\cot (\angle S)-\cot (\angle R)}, \quad A_{z}=\frac{\overline{S R}}{\cot (\angle S)-\cot (\angle R)} .
$$

To employ the ray geometry of different source-receiver gathers and to substitute (1) and (2) into (3), I determine the reflected points explicitly as follows.

\subsubsection{A common shot gather}

Let the offset $(\overline{S R})$ in a common shot gather be denoted by $x$ and the distance from the source to the interface be $h$ (Fig. 1a). The offset for the station ( $\left.S^{\prime \prime}\right)$ located above the virtual source and that receiving a vertical shooting ray are

$$
x_{m s}=-2 h \sin \alpha \text { and } x_{n s}=2 h \sin \alpha / \cos 2 \alpha,
$$

respectively. By applying (1) to the case of a common shot gather, the direction of an inverse ray emitting from the receiver is determined,

$$
\cos (\angle R)=\frac{x_{m s}-x}{V t_{x}}, \quad \sin (\angle R)=\frac{2 h \cos \alpha}{V t_{x}} .
$$

Similarly, from (2) and $\overline{R R^{\prime}}=2(h+x \sin \alpha)$ in Fig. 1a, the direction of an inverse ray shooting from the source is obtained,

$$
\cos (\angle S)=\cos 2 \alpha \frac{x-x_{n s}}{V t_{x}}, \quad \sin (\angle S)=2 \sin \alpha \frac{h+x \sin \alpha}{V t_{x}} .
$$

Equations (5) and (6) indicate that an inverse ray of a common shot gather emits from the receiver with a negative $\mathrm{x}$ component of the direction when $x>x_{m s}$ and it shoots from the source with a negative direction cosine when $x<x_{n s}$ (the case of Fig. 1a). Finally, to substitute (5) and (6) into (3), the reflected point is derived in a closed form,

$$
A_{x}=h \cos 2 \alpha \frac{x-x_{n s}}{2 h+x \sin \alpha}, \quad A_{z}=2 h \cos \alpha \frac{h+x \sin \alpha}{2 h+x \sin \alpha} .
$$

Equation (7) is independent of $V$ while $h$ and $\alpha$ in (7) can be determined from fitting the travel-time hyperbola (A3) through the least travel-time error, as indicated in Appendix A.

\subsubsection{A common midpoint gather}

Let the offset $(\overline{S R})$ in a common midpoint gather be denoted by $x$ and the distance from the midpoint ( $M$ in Fig. 1b) to the interface be $h$. By considering the geometry of the ray shooting (or receiving as the solid arrow) vertically from $M_{1}$ to $D$ and receiving (or shooting as the solid arrow) at $M_{2}$ in Fig. 1b, the offset between $M_{1}$ and $\dot{M}_{2}$ is

$$
x_{m m}=2 h \sin \alpha /(\cos \alpha)^{2},
$$


where $\overline{M_{1} D}=\left(h-x_{m m} \sin \alpha / 2\right) \sec \alpha$ and $x_{m m}=\overline{M_{1} D} \tan 2 \alpha$ are considered. According to (1) and $\overline{S S^{\prime}}=2(h-x \sin \alpha / 2)$ in Fig. 1b, the direction of an inverse ray from the receiver of a common midpoint gather is determined,

$$
\cos (\angle R)=\frac{x+x_{m m}}{V t_{x}}(\cos \alpha)^{2}, \quad \sin (\angle R)=\frac{2 h-x \sin \alpha}{V t_{x}} \cos \alpha .
$$

Similarly, in view of (2) and $\overline{R R^{\prime}}=2(h+x \sin \alpha / 2)$ in Fig. 1b, the direction of an inverse ray from the source is obtained,

$$
\cos (\angle S)=\frac{x-x_{m m}}{V t_{x}}(\cos \alpha)^{2}, \quad \sin (\angle S)=\frac{2 h+x \sin \alpha}{V t_{x}} \cos \alpha .
$$

Equations (9) and (10) indicate that an inverse ray of a common midpoint gather emits from the receiver with a negative $x$ component of the direction when $x>-x_{m m}$, and it shoots from the source with a positive direction cosine when $x>x_{m m}$ (the case of Fig. 1b). Finally, substituting (9) and (10) into (3), the reflected point with respect to the common midpoint is derived in a closed form,

$$
A_{x}=-h \sin \alpha\left(1+\left(\frac{x \cos \alpha}{2 h}\right)^{2}\right), \quad A_{z}=h \cos \alpha\left(1-\left(\frac{x \sin \alpha}{2 h}\right)^{2}\right) .
$$

Unfortunately, since the travel-time hyperbola of a common midpoint gather $t_{x}^{2}=(2 h / V)^{2}+(x \cos \alpha / V)^{2}$ is symmetrical with respect to $x$, parameters $h$ and $\alpha$ in (11) can be determined from fitting the travel-time hyperbola only if $V$ is known in advance. Furthermore, the square of $\cos \alpha$ in the travel-time hyperbola results in the uncertain sign of $\sin \alpha$ in (11) for structural imaging.

\subsubsection{A common offset gather}

Let the offset $(\overline{S R})$ in a common offset gather be denoted by $x^{*}$ and the source location, the distance from the intersection of the interface and the surface $(T)$ to the source, be $x$, as shown in Fig. 1c. I observe that the source locations for two special cases of shooting

$\left(x^{*}>\right.$ $0)$ and receiving $\left(x^{*}<0\right)$ a vertical ray (Fig. 2$)$ are

$$
x_{n o}=\frac{\cos 2 \alpha}{2(\sin \alpha)^{2}} x^{*} \text { and } x_{m o}=-\frac{x^{*}}{2(\sin \alpha)^{2}},
$$

respectively. By considering (1) and $\overline{S S^{\prime}}=2 x \sin \alpha$ in Fig. 1c, the direction of an inverse ray from the receiver of a common offset gather is determined,

$$
\cos (\angle R)=2(\sin \alpha)^{2} \frac{x_{m o}-x}{V t_{x}}, \sin (\angle R)=\frac{x \sin 2 \alpha}{V t_{x}} .
$$

Similarly, from (2) and $\overline{R R^{\prime}}=2\left(x+x^{*}\right) \sin \alpha$ in Fig. 1c, the direction of an inverse ray from 


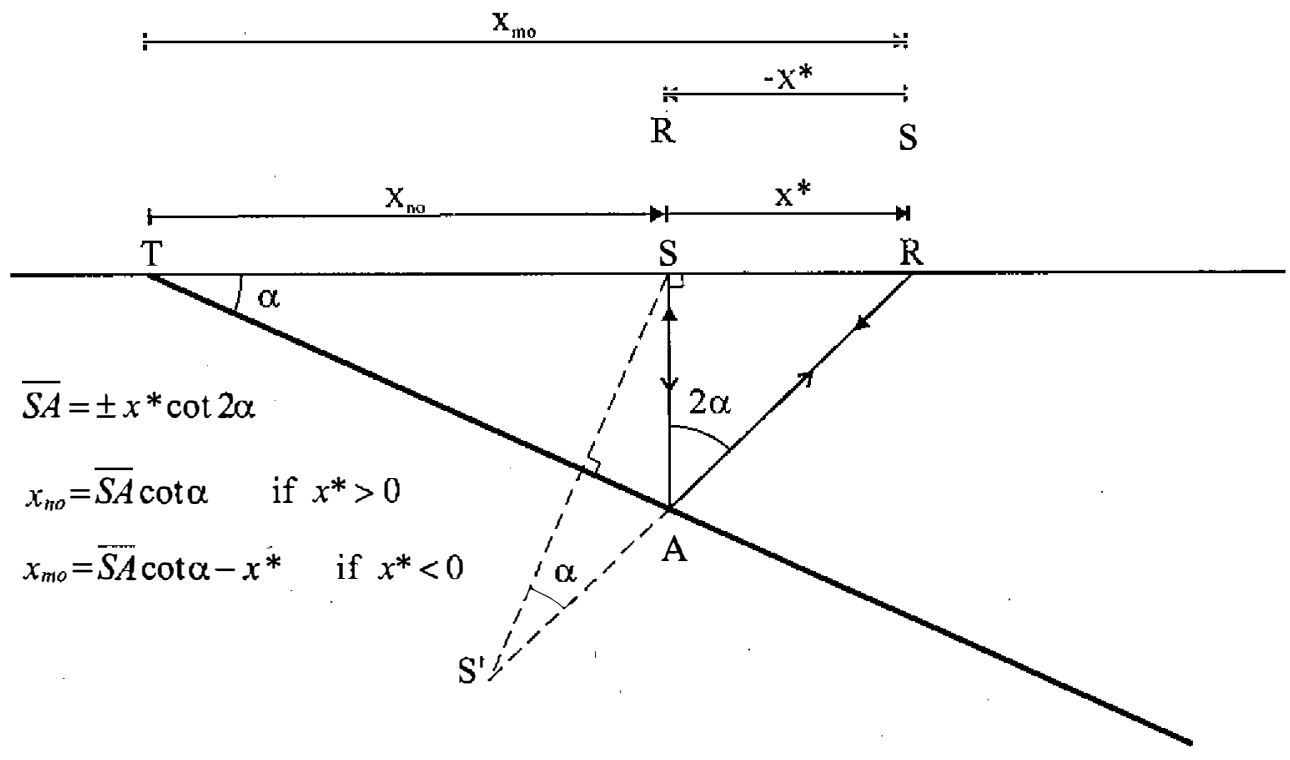

Fig. 2. Two special cases of shooting $\left(\mathrm{x}^{*}>0\right)$ and receiving $\left(\mathrm{x}^{*}<0\right)$ a vertical ray in a common offset gather. The length labels of $x_{n o}$ and $x^{*}$ are for the former case while the solid arrows and those of $x_{m o}$ and $-x^{*}$ are for the latter case.

the source is obtained,

$$
\cos (\angle S)=2(\sin \alpha)^{2} \frac{x_{n o}+x}{V t_{x}}, \quad \sin (\angle S)=\frac{\left(x+x^{*}\right) \sin 2 \alpha}{V t_{x}} .
$$

Equations (13) and (14) indicate that an inverse ray of a common offset gather emits from the receiver with a negative $x$ component of the direction when $x>x_{m o}$ and it shoots from the source with a positive direction cosine when $x<x_{n o}$ (the case of Fig. 1c). Finally, substituting (13) and (14) into (3), the reflected point with respect to the intersection of the interface and the surface $(T)$ is derived in a closed form,

$$
A_{x}=2(\cos \alpha)^{2} \frac{x\left(x+x^{*}\right)}{2 x+x^{*}}, \quad A_{z}=\sin 2 \alpha \frac{x\left(x+x^{*}\right)}{2 x+x^{*}} .
$$

The dip of the interface $\alpha$ in (15) can be determined from fitting the travel-time hyperbola of a common offset gather through the least travel-time error, as similar to Appendix A. Equation (15) also indicates that the slope of the reflected point $A_{z} / A_{x}$ equals the slope of the interface that supports the accuracy of the prestack inverse-ray imaging. Furthermore, the poststack inverse-ray imaging (May and Covey 1981) can be derived from (13)-(15) for the case of the zero offset $\left(x^{*}=0\right)$.

\subsection{Elliptic Envelope}

Let the distance between a source and a receiver be $2 c$. A reflected point $A$ with the travel- 
time $t$ should be located at an ellipse with the foci at $x= \pm c$, and its semi-major and semiminor axes should be $a=V t / 2$ and $b=\sqrt{a^{2}-c^{2}}$, respectively (Fig. 3). If the layer velocity $(V)$ is known, an envelope of two reflected ellipses in three types of source-receiver gather is investigated for determining the reflected points $(a \cos \theta, b \sin \theta)$ or the angle $\theta$.

\subsubsection{A common shot gather}

Let $x_{1}$ and $x_{2}$ be the half offsets of two receivers in a common shot acquisition (Fig. 4a). Since two reflected points at two reflected ellipses of a common shot gather are

$$
A_{1}=\left(x_{1}+a_{1} \cos \theta_{1}, b_{1} \sin \theta_{1}\right) \text { and } A_{2}=\left(x_{2}+a_{2} \cos \theta_{2}, b_{2} \sin \theta_{2}\right) \text {, }
$$

their parallel tangents (B2) at the elliptic envelope imply that

$$
\frac{a_{1}}{b_{1}} \tan \theta_{1}=\frac{a_{2}}{b_{2}} \tan \theta_{2}
$$

Similarly, since the slope of the vector $\overrightarrow{A_{1} A_{2}}$ is equal to the slope of $d A / d \theta(\mathrm{B} 2)$, I obtain

$$
\frac{b_{2} \sin \theta_{2}-b_{1} \sin \theta_{1}}{x_{2}-x_{1}+a_{2} \cos \theta_{2}-a_{1} \cos \theta_{1}}=-\frac{b_{i}}{a_{i}} \cot \theta_{i}
$$

Substituting (18) into (17) leads to

$$
\frac{a_{\mathrm{j}}}{\cos \theta_{1}}-\frac{a_{2}}{\cos \theta_{2}}=x_{2}-x_{1}
$$

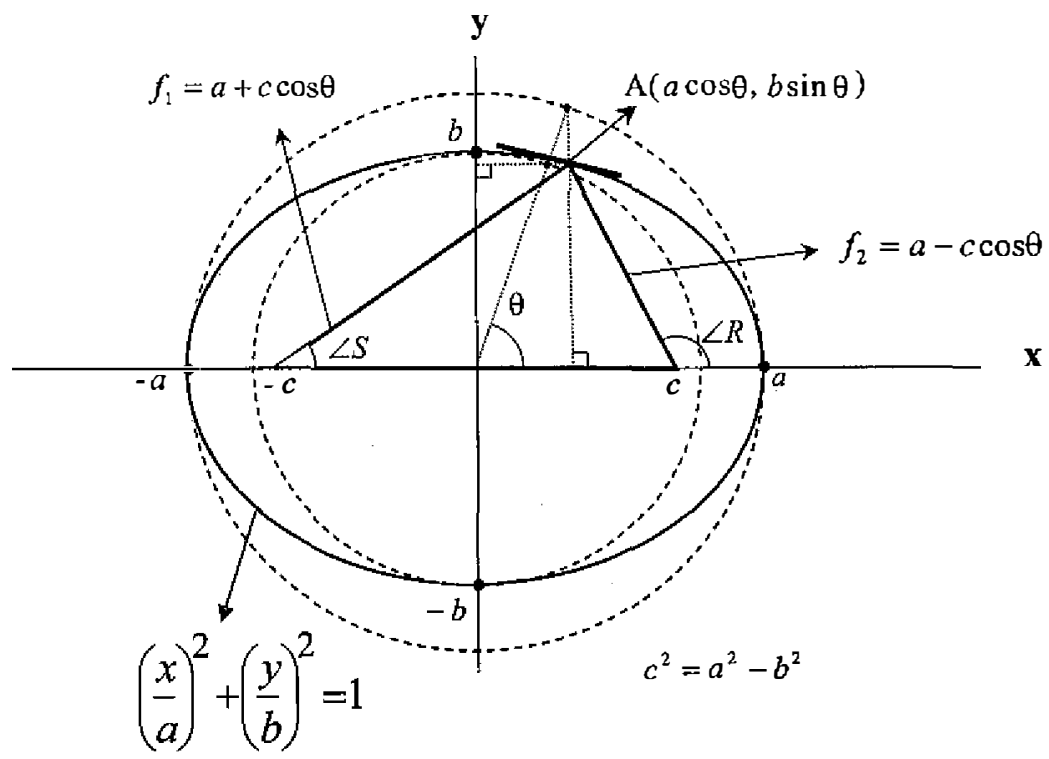

Fig. 3. Properties of a reflected ellipse. A reflected point and its tangent are displayed as $A$ and a thick line, respectively. 


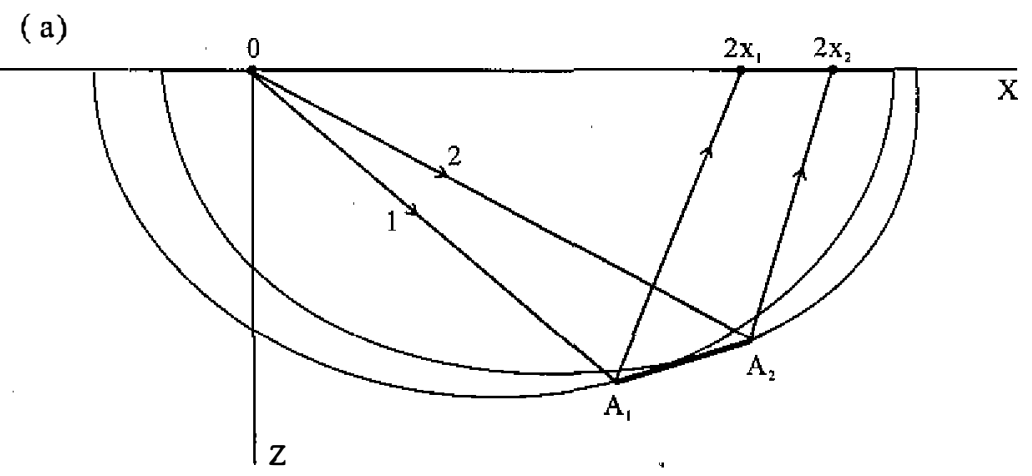

(b)

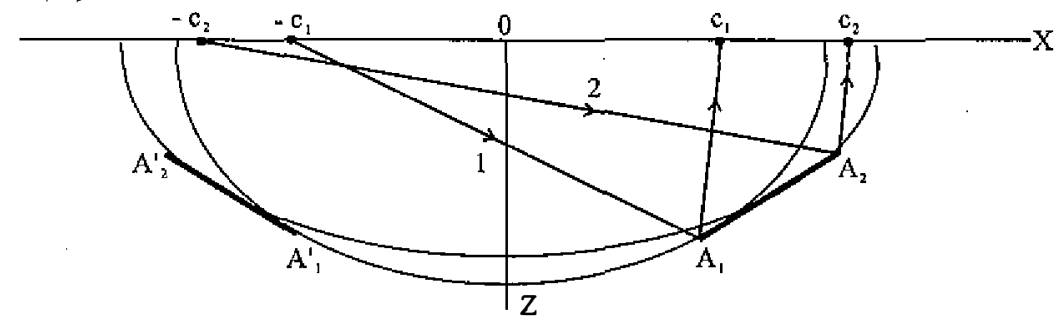

(c)

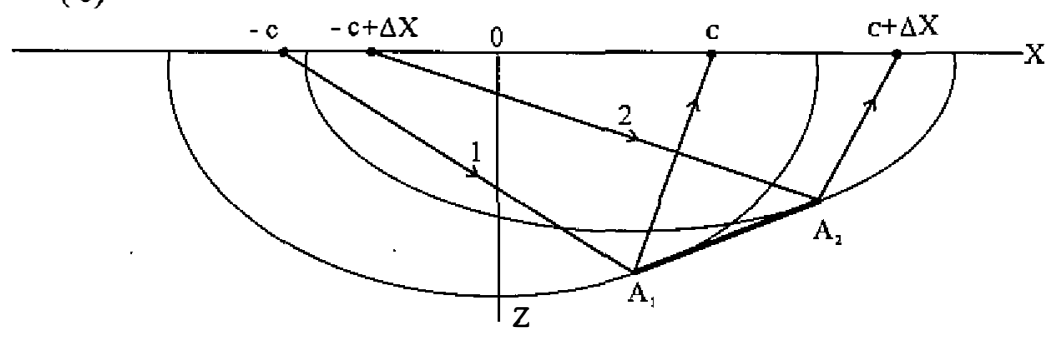

Fig. 4. An envelope of two reflected ellipses in (a) a common shot gather, (b) a common midpoint gather, and (c) a common offset gather. A thick line $\overline{A_{1}^{\prime} A_{2}^{\prime}}$ in (b) is another elliptic envelope in the common midpoint gather.

Therefore, the angle $\theta_{i}$ can be determined from (17) and (19),

$$
\sec \theta_{i}=\frac{b_{i}^{2}\left(x_{2}-x_{1}\right)+b_{1}^{2} x_{2}-b_{2}^{2} x_{1}}{a_{i}\left(b_{1}^{2}-b_{2}^{2}\right)}, i=1,2
$$

where two roots from $\pm \sqrt{b_{1}^{2}} b_{2}^{2} \overline{\left(x_{2}-x_{1}\right)^{2}+\left(b_{1}^{2}-b_{2}^{2}\right)\left(b_{1}^{2} a_{2}^{2}-a_{1}^{2} b_{2}^{2}\right)}$ in (20) has been se- 
lected as $b_{1}^{2} x_{2}-b_{2}^{2} x_{1}$ since $b_{2}^{2} x_{1}-b_{1}^{2} x_{2}$ will result in the contrary result $\left|\sec \theta_{i}\right|=c / a_{i}<1$. Reflected points and directions of two inverse rays can be derived from substitution of $(20)$ in (16) and (B5)-(B6), respectively.

\subsubsection{A common midpoint gather}

Since two reflected points at two reflected ellipses of a common midpoint gather (Fig. 4b) are

$$
A_{i}=\left(a_{i} \cos \theta_{i}, b_{i} \sin \theta_{i}\right),
$$

(17) can be applied but (19) is rewritten as

$$
\frac{a_{\mathrm{I}}}{\cos \theta_{1}}=\frac{a_{2}}{\cos \theta_{2}} \text {. }
$$

To consider (17) and (22), I find

$$
\sec ^{2} \theta_{i}=\frac{b_{1}^{2} a_{2}^{2}-a_{1}^{2} b_{2}^{2}}{a_{i}^{2}\left(b_{1}^{2}-b_{2}^{2}\right)}, i=1,2 .
$$

Therefore, two reflected points are obtained by substituting (23) into (21),

$$
A_{i}=\left( \pm a_{i}^{2} \sqrt{\frac{b_{1}^{2}-b_{2}^{2}}{b_{1}^{2} a_{2}^{2}-a_{1}^{2} b_{2}^{2}}}, b_{i}^{2} \sqrt{\frac{a_{2}^{2}-a_{1}^{2}}{b_{1}^{2} a_{2}^{2}-a_{1}^{2} b_{2}^{2}}}\right), i=1,2 .
$$

The effect of the double root in the $\mathrm{x}$ component of the reflected points (24) can also be seen as $\overline{A_{1} A_{2}}$ and $\overline{A_{1}^{\prime} A_{2}^{\prime}}$ in Fig. 4b, which is similar to the uncertain sign of $\sin \alpha$ in the method of ray geometry.

\subsubsection{A common offset gather}

Let the shot interval or the receiver interval of a common offset gather $\left(c=c_{1}=c_{2}\right)$ be $\Delta X$, then two reflected points at two reflected ellipses are

$$
A_{1}=\left(a_{1} \cos \theta_{1}, b_{1} \sin \theta_{1}\right), A_{2}=\left(\Delta X+a_{2} \cos \theta_{2}, b_{2} \sin \theta_{2}\right) .
$$

Equation (17) is still applied but (19) is rewritten as

$$
\frac{a_{\mathrm{I}}}{\cos \theta_{1}}-\frac{a_{2}}{\cos \theta_{2}}=\Delta X
$$

To consider (17) and (26), I find

$$
\sec \theta_{i}=\frac{b_{i}^{2} \Delta X+\sqrt{b_{1}^{2} b_{2}^{2} \Delta X^{2}+c^{2}\left(b_{1}^{2}-b_{2}^{2}\right)^{2}}}{a_{i}\left(b_{1}^{2}-b_{2}^{2}\right)}, i=1,2 .
$$

The plus sign of the square root is selected in (27) since the absolute value of this result is larger than that with the minus sign. Furthermore, for the case of the zero offset $\left(c=0, a_{i}=b_{i}\right)$, 
(27) can be reduced to

$$
\sec \theta_{1}=\sec \theta_{2}=\frac{\Delta X}{b_{1}-b_{2}},
$$

which is equivalent to the take-off angle for propagation of the poststack inverse rays.

Analysis of the ray geometry and the elliptic envelope indicates that, due to the symmetry of the travel-time hyperbola and the double root of the reflected points, a common midpoint gather is not suitable for determining the structural velocity and interface. On the other hand, a common shot gather and a common offset gather can be applied to image the layer velocity and the structural interface. In particular, from a common offset gather, the poststack inverse ray is proved as a special case of the prestack inverse ray.

\section{RESULTS AND DISCUSSIONS}

Reflected rays traveling in 2D sedimentary structures with a P-wave velocity of $3 \mathrm{~km} / \mathrm{s}$ and the associated travel times are first calculated by using the stable-beam method (Wang and Waltham 1995). The ray geometry (equation (7)) and the elliptic envelope (equations (16) and (20)) of the common shot gathers are subsequently applied to evaluate the errors of inverse rays and to image a sedimentary basin.

\subsection{Error Analysis}

A flat interface with a depth of $500 \mathrm{~m}$ is used to test the accuracy of the prestack inverse rays subject to the limited number and the uncertainty of travel-time picks. By applying the minimum number (three) of travel-time picks with an uncertainty of $20 \mathrm{~ms}$ to the ray geometry, inverse rays of a common shot gather (the inset of Fig. 5) are constructed. The travel-time uncertainty is chosen because the wavelet length is designed about $20 \mathrm{~ms}$ in most of the MCS surveys. The depth errors of reflected points from the ray geometry, indicated by the circles in Fig. 5, are much greater and more unstable at far offsets (offsets $>1100 \mathrm{~m}$ ) than those at near offsets. On the other hand, the depth errors of the prestack inverse-ray imaging based on the elliptic envelope, indicated by the triangles in Fig. 5, are slightly increasing from $30 \mathrm{~m}$ at the zero offset to about $70 \mathrm{~m}$ at the offset of $2000 \mathrm{~m}$. The large error of ray geometry at far offsets result from the insufficient travel-time picks for determining the layer velocity and for extrapolating the shortest travel-time and offset pairs of the reflected hyperbola according to Appendix A. Therefore, when the travel-time picks of a reflected hyperbola is limited as the extreme case in Fig. 5, the method of ray geometry is recommended for applying at near offsets where the results from both methods are similar. Alternatively, the method of elliptic envelope is suitable for imaging at far offsets where the results from both methods show great differences.

Prestack inverse-ray imaging over interfaces with dips of $30^{\circ}, 45^{\circ}$ and $60^{\circ}$ (insets of Fig. 6) are investigated for testing the imaging errors subject to the dips of the interfaces and the uncertainty of the travel-time picks. The depth errors of three dip models (Fig. 6) show that the increasing dips of the interfaces increase the errors of both methods. Furthermore, the method 


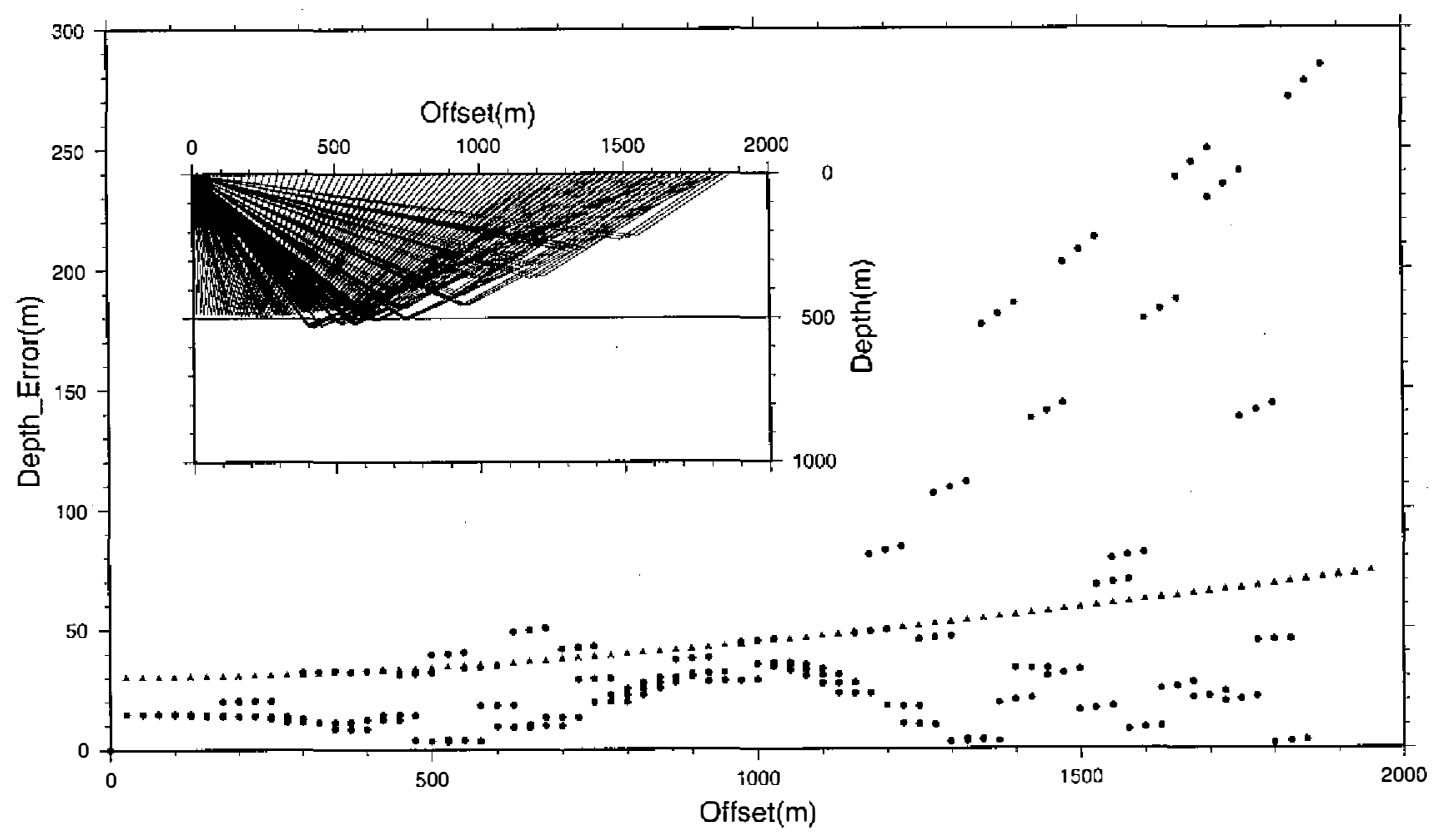

Fig. 5. Errors of the prestack inverse-ray imaging over a flat interface. The circles and the triangles denote the errors subject to a travel-time uncertainty of $20 \mathrm{~ms}$ by considering the geometry of three reflected rays and the envelope of two reflected ellipses, respectively. Inverse rays traveling in the flat layer by using the method of ray geometry are shown in the inset.

of elliptic envelope (triangular symbols) is unstable at far offsets and for imaging shallow interfaces. The instability from the method of elliptic envelope may be attributed to the influence of the travel-time uncertainty at the denominator in (20). Therefore, the method of ray geometry is, in general, superior to the method of elliptic envelope when the travel-time picks are sufficient.

\subsection{Imaging of a Sedimentary Basin}

Prestack inverse-ray imaging is applied on a sandbox model of a strike-slip fault and pullapart basin (Wang and McClay 1995). For constructing a 2D homogeneous layer from the inverse rays, I select the top layer of the sandbox model and a longitudinal section through the center of the model. The reflected travel-times from six shots with a shot spacing of $2 \mathrm{~km}$ and 25 receivers with a receiver interval of $0.15 \mathrm{~km}$ for each shot are computed by using the stablebeam method (Fig. 7). The acquisition in this seismic simulation is sparse, compared to that required in the conventional MCS survey, in order to test the capability of the prestack inverse-ray imaging. I observe that first arrivals in travel-time curves of the second and fifth shots are limited (Fig. 7), which implies the arrival terminations due to the structural kinks. Furthermore, picks of the later arrivals in the first two shots are difficult because lots of them 


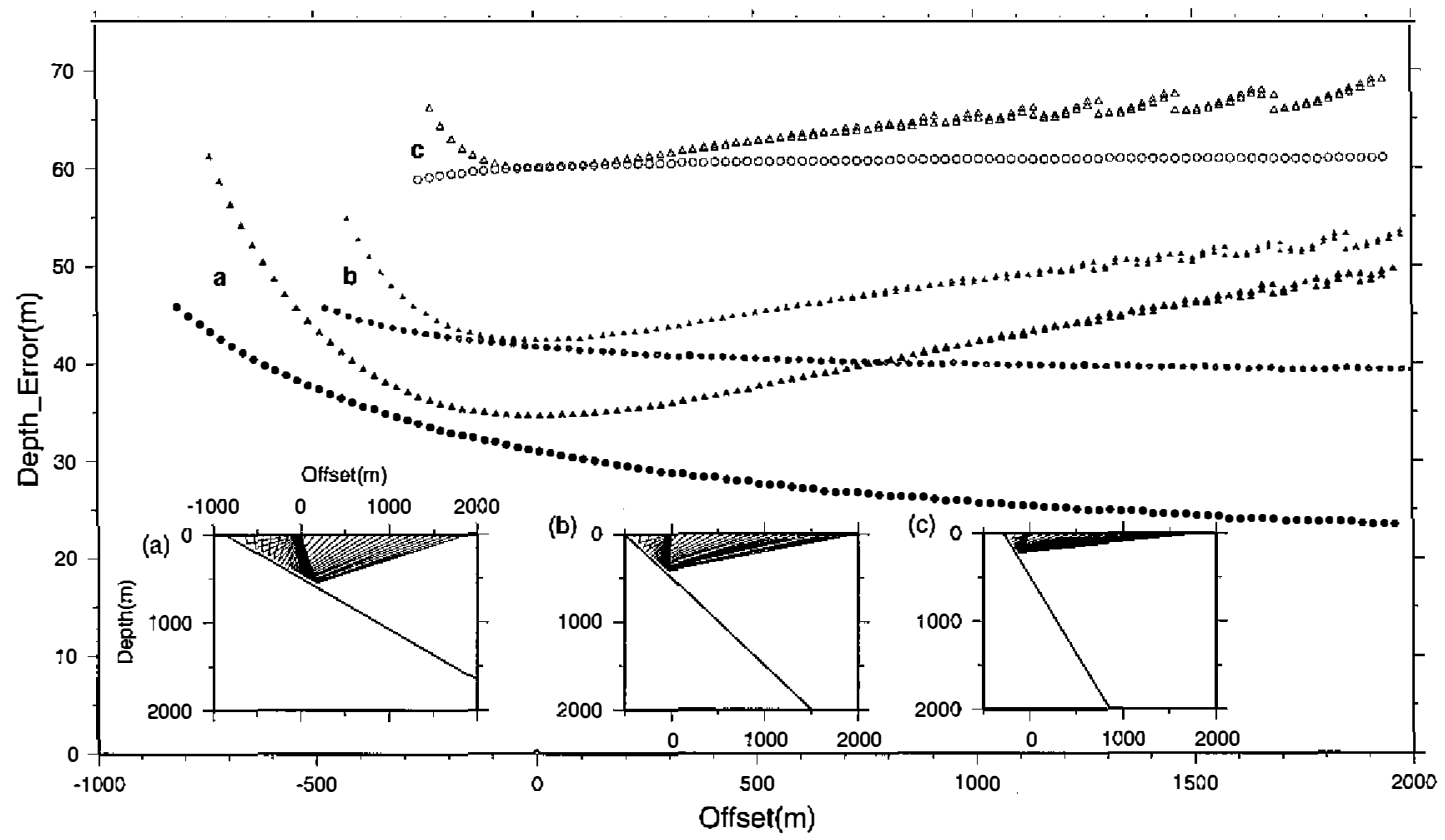

Fig. 6. Errors of the prestack inverse-ray imaging over interfaces with dips of (a) $30^{\circ}$, (b) $45^{\circ}$ and (c) $60^{\circ}$. The circles and the triangles denote the errors subject to a ravel-time uncertainty of $20 \mathrm{~ms}$ by using the methods of ray geometry and elliptic envelope, respectively. Inverse rays traveling in three dip interfaces by using the method of elliptic envelope are shown in the insets.

are close to each other. In particular, arrivals later than $5 \mathrm{~s}$ in the third shot gather may not be seen in the real data.

For the prestack inverse-ray imaging, each travel-time hyperbola must be picked separately (e.g. the first arrivals in the last shot) since the layer velocity $(V)$, the distance from a source to an interface $(h)$ and the dip of the interface $(\alpha)$ are evaluated from each ravel-time hyperbola according to Appendix A. The results of the prestack inverse-ray imaging show that the first arrivals only constrain the shallow interface as shown by inverse rays as dark lines and reflected points as dark dots in Fig. 8. The interface imaged by ray geometry (dark dots) and that constructed from elliptic envelope (dark lines) are similar except when rays emit from the second and fifth shots. The differences can be attributed to large errors of $V, h$ and $\alpha$ from the method of ray geometry when arrivals at a travel-time hyperbola are limited. Imaging by the later arrivals (gray lines and cross symbols in Fig. 8), especially from the first two shots, fill up the deep interface and other portions that lack constraints from the first arrivals. The method of ray geometry still generates the errors due to few arrivals at the travel-time hyperbola in the first, second, fifth and sixth shots.

The imaging error of a sedimentary basin when using the elliptic envelope (Fig. 8) is 


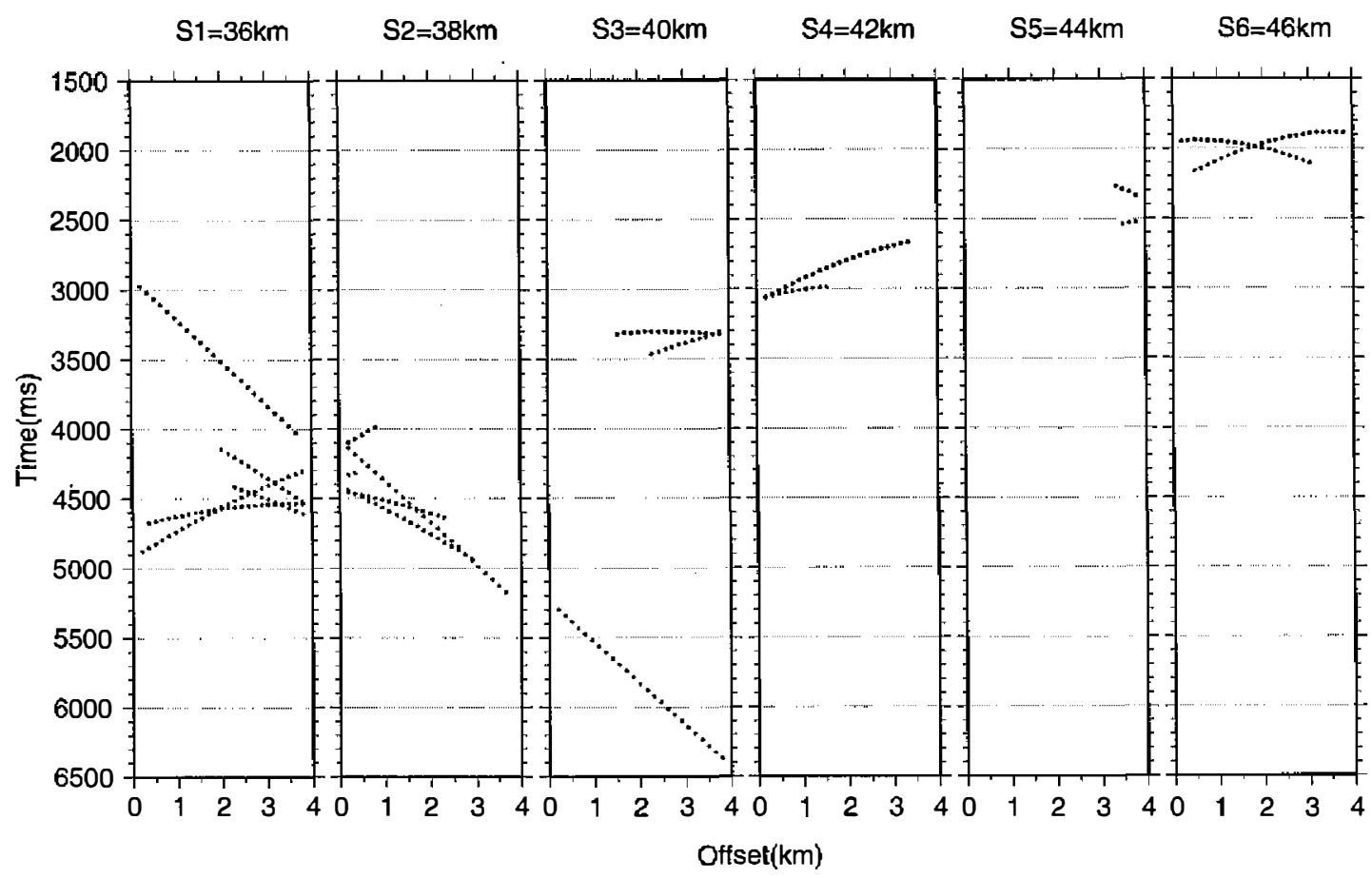

Fig. 7. Reflected travel-times in six common shot gathers of a sedimentary basin. Travel-time picks as dark and gray dots are, respectively, the first and later arrivals used for prestack inverse-ray imaging of the basin.

small compared to those in the flat and dip layers (Figs. 5 and 6) because the depth of the basin is greater than the extreme cases in the error analysis. Hence, the prestack inverse-ray imaging is better when applied to the deep seismic survey. Applications of the prestack inverse-ray imaging also suggest that the layer velocity can be determined from ray geometry (Appendix A), while the method of elliptic envelope requires the layer velocity in advance. Furthermore, the method of ray geometry is more accurate than the method of elliptic envelope (Fig. 6), except when the travel-time hyperbola possesses limited arrivals (Figs. 5 and 8). For the latter case, the method of elliptic envelope is recommended at far offsets where the results from both methods show great differences. Therefore, velocity determination from ray geometry and interface imaging by considering both methods will be the practical way for prestack inverseray imaging.

Similar to the applications of common shot gathers presented in this section, prestack inverse-ray imaging can be readily applied to common offset gathers. Furthermore, since the arrival terminations seen in the above example imply the structural kinks, prestack inverse-ray imaging with diffracted arrivals is possible by considering the intersection of diffracted ellipses. For the case of 3D multi-layers, the envelopes of reflected ellipsoids (Pivot 1997) and the intersection of diffracted ellipsoids can be considered to image each layer at a time by using 


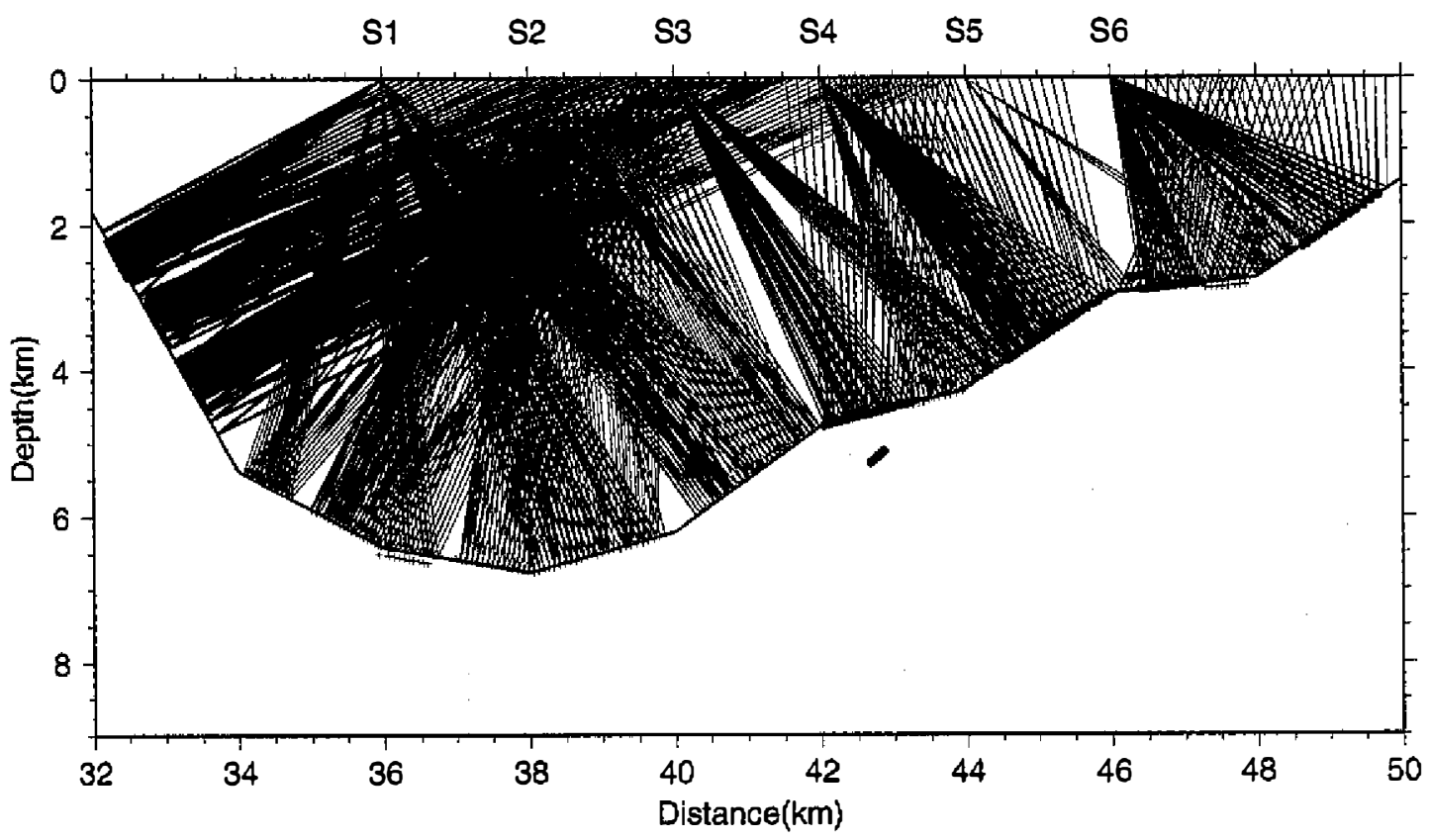

Fig. 8. Prestack inverse-ray imaging of a sedimentary basin. Reflected rays as dark and gray lines are derived from the method of elliptic envelope with the first and later arrivals, respectively. Reflected points as solid circles and cross symbols are obtained from the method of ray geometry with the first and later arrivals, respectively. Solid lines at the bottom are the true interface.

travel-time picks from all layers (Wang and Tan 2002). However, the prestack inverse-ray imaging of inhomogeneous media is still one of the unsolved issues that need to be further investigated.

\section{CONCLUSIONS}

By considering the geomery of reflected rays from image sources and image receivers in a common shot gather, a common midpoint gather and a common offset gather, I determine the layer velocity and two directions of an inverse ray (one emitting from a source and another receiving by a station) traveling in a $2 \mathrm{D}$ homogeneous layer. An envelope of reflected points with a constant travel-time in three types of source-receiver gathers is also investigated for prestack inverse-ray imaging of a 2D homogeneous irregular layer. Although the structural velocity, dip and depth can be derived purely from fitting of the travel-time hyperbola, the inverse rays provide full coverage of the constrained images additionally. Applications of the prestack inverse rays to the error analysis and to imaging a sedimentary basin suggest that the method of ray geometry is, in general, superior to the method of elliptic envelope, except when 
the travel-time hyperbola possesses limited arrivals. Therefore, the best way to apply prestack inverse rays is to determine the layer velocity from ray geometry and to image the interface by considering both methods.

Results from this study can be used to rapidly and accurately process multi-channel seismic data with a low signal-to-noise ratio in the fields of engineering geology (e.g. fracture surfaces in the tunnel and land slide), seismology (e.g. normal and thrust fault planes), resource exploration (e.g. salt and shale) and plate tectonics (e.g. subduction complex and fracture zone). Therefore, the prestack inverse-ray imaging offers great potential for applications in migration velocity analysis and real-time monitoring.

Acknowledgements I would like to thank Ching-Hsiang Hsu (CPC) and an anonymous reviewer for their suggestions to improve this paper. Cheng-Sung Wang (IAG/NTOU) is also acknowledged for his encouragement to submit this paper to TAO. The idea of inverse-ray imaging was initiated through discussions with my former students S. C. Tan and K. C. Chang whose studies were supported by NSC 89-2611-M-019-011 and NSC 88-2611-M-019-014ODP, respectively. The theory of this paper was originally prepared for a NSC proposal to the current and upcoming projects of NSC 91-2611-M-019-006 and NSC 92-2611-M-019-001. Praise the LORD to let this work done soon.

\section{REFERENCES}

Donihoo, K., N. Bernitsas, N. Dai, G. Martin, and D. Shope, 2001: Is depth imaging a commodity? The impact of new imaging technologies and web-based collaboration. The Leading Edge, 20, 486-496.

Gray, S. H., J. Etgen, J. Dellinger, and D. Whitmore, 2001: Seismic migration problems and solutions. Geophysics, 66, 1622-1640.

Hua, B. L., and G. A. McMechan, 2001: Parsimonious 2-D poststack Kirchhoff depth migration. Geophysics, 66, 1497-1503.

May, B. T., and J. D. Covey, 1981: An inverse ray method for computing geologic structures from seismic reflections - Zero-offset case. Geophysics, 46, 268-287.

Operto, M. S., S. Xu, and G. Lambare, 2000: Can we quantitatively image complex structures with rays? Geophysics, 65, 1223-1238.

Pivot, F., 1997: A geometrical method for migrating 3-D or 2.5-D seismic reflection data. Geophys. Res. Lett., 24 , 3321-3324.

Raynaud, B., 1988: A 2-D, ray-based, depth migration method for deep seismic reflections. Geophys. J., 93, 163-171.

Wang, T. K., and K. R. McClay, 1995: Gaussian beam seismic modeling and processing of complex 3-D structures. 65 $5^{\text {th }}$ Ann. Internat. Mtg., Soc. Expl. Geophys., Expanded Abstracts, 552-555.

Wang, T. K., and S. C. Tan, 2003: Inverse-ray imaging of 2D layered structures from seismic reflection data. Chinese J. Mechanics, accepted.

Wang, X., and D. A. Waltham, 1995: The stable-beam seismic modelling method. Geophysi- 
cal Prospecting, 43, 939-961.

Warner, M., 1987: Migration - why doesn't it work for deep continental data? Geophys. J. R. astr. Soc., 89, 21-26.

\section{APPENDIX A}

\section{A Travel-Time Hyperbola of a Common Shot Gather}

By considering the cosine theorem of the triangle SS'R in Fig. 1a, I have

$$
{\overline{S^{\prime} R}}^{2}=4 h^{2}+x^{2}+4 h x \sin \alpha .
$$

A travel-time hyperbola of a common shot gather can be derived from dividing (A1) by the square of the layer velocity,

$$
t_{x}^{2}=t_{m}^{2}+\left(\frac{x-x_{m s}}{V}\right)^{2},
$$

where the shortest travel-time and offset pairs of the reflected hyperbola are

$$
t_{m}=\frac{2 h}{V} \cos \alpha \text { and } x_{m s}=-2 h \sin \alpha,
$$

respectively. The receiver with the offset $x_{m s}$ is located above the virtual source (S').

Since the shortest travel-time $\left(t_{m}\right)$ and the associated offset $\left(x_{m s}\right)$ in general cannot be picked from the travel-time hyperbola (A2) in a common shot section, the least travel-time error is applied to fit the travel-time hyperbola and to determine $V, x_{m s}$ and $t_{m}$. Let the square of the travel-time error be

$$
E=\sum_{i=1}^{N}\left(a x_{i}^{2}+b x_{i}+c-t_{i}^{2}\right)^{2},
$$

where $a=V^{-2}, b=-2 x_{m s} / V^{2}, c=\left(t_{m}\right)^{2}+\left(x_{m s} / V\right)^{2}$ and $N$ is the number of the travel-time picks. If the derivatives of $E$ with respect to $a, b$ and $c$ are zero, I derive

$$
\left[\begin{array}{lll}
S_{4} & S_{3} & S_{2} \\
S_{3} & S_{2} & S_{1} \\
S_{2} & S_{1} & S_{N}
\end{array}\right]\left[\begin{array}{c}
V^{-2} \\
-2 x_{m s} / V^{2} \\
t_{m}^{2}+\left(x_{m s} / V\right)^{2}
\end{array}\right]=\left[\begin{array}{c}
B_{2} \\
B_{1} \\
B_{0}
\end{array}\right],
$$

where

$$
S_{n}=\sum_{i=1}^{N} x_{i}^{n}, n=1,2,3,4 \text { and } B_{m}=\sum_{i=1}^{N}\left(x_{i}^{m} t_{i}^{2}\right), m=0,1,2
$$

By considering the matrix inversion, (A5) is reduced to 


$$
\left[\begin{array}{c}
V^{-2} \\
-2 x_{m s} / V^{2} \\
t_{m}^{2}+\left(x_{m s} / V\right)^{2}
\end{array}\right]=\frac{1}{D_{0}}\left[\begin{array}{lll}
C_{1} & C_{4} & C_{6} \\
C_{4} & C_{2} & C_{5} \\
C_{6} & C_{5} & C_{3}
\end{array}\right]\left[\begin{array}{c}
B_{2} \\
B_{1} \\
B_{0}
\end{array}\right]
$$

where

$$
\begin{aligned}
& D_{0}=N S_{2} S_{4}+2 S_{1} S_{2} S_{3}-N S_{3}^{2}-S_{1}^{2} S_{4}-S_{2}^{3} \neq 0, \\
& C_{1}=N S_{2}-S_{1}^{2}, C_{2}=N S_{4}-S_{2}^{2}, C_{3}=S_{2} S_{4}-S_{3}^{2}, \\
& C_{4}=S_{1} S_{2}-N S_{3}, C_{5}=S_{2} S_{3}-S_{1} S_{4}, C_{6}=S_{1} S_{3}-S_{2}^{2} .
\end{aligned}
$$

Therefore, the layer velocity, the offset and the shortest travel-time,

$$
V=\sqrt{\frac{D_{0}}{D_{1}}}, x_{m s}=-\frac{D_{2}}{2 D_{1}} \text { and } t_{m}=\sqrt{\frac{4 D_{1} D_{3}-D_{2}^{2}}{4 D_{0} D_{1}}},
$$

are obtained, where

$$
\begin{aligned}
& D_{1}=C_{1} B_{2}+C_{4} B_{1}+C_{6} B_{0}, \\
& D_{2}=C_{4} B_{2}+C_{2} B_{1}+C_{5} B_{0}, \\
& D_{3}=C_{6} B_{2}+C_{5} B_{1}+C_{3} B_{0} .
\end{aligned}
$$

The distance from a source to an interface $(h)$ and the dip of the interface $(\alpha)$ can be readily derived from substitution of (A6) and (A8)-(A14) into (A3).

\section{APPENDIX B}

\section{A Reflected Ellipse}

Since a reflected point $A$ at an ellipse of a constant travel time can be indicated as

$$
A=(a \cos \theta, b \sin \theta),
$$

the tangential vector of the reflected ellipse at $A$ is

$$
\frac{d A}{d \theta}=(-a \sin \theta, b \cos \theta) .
$$

The angle $\theta$ in the reflected ellipse can be drawn from projecting the reflected point onto two circles (dashed lines in Fig. 3) with radii of $a$ and $b$ along the directions of the minor and major axes, respectively. On the other hand, by considering $b^{2}=a^{2}-c^{2}$ and the reflected ellipse (Fig. 3), distances between the reflected point and the foci are

$$
\begin{aligned}
& f_{1}=\sqrt{(a \cos \theta+c)^{2}+b^{2} \sin ^{2} \theta}=a+c \cos \theta, \\
& f_{2}=\sqrt{(a \cos \theta-c)^{2}+b^{2} \sin ^{2} \theta}=a-c \cos \theta .
\end{aligned}
$$


Therefore, directions of an inverse ray emitting from the foci of a reflected ellipse (Fig. 3) can be derived from

$$
\begin{array}{ll}
\cos (\angle S)=\frac{a \cos \theta+c}{a+c \cos \theta}, & \sin (\angle S)=\frac{b \sin \theta}{a+c \cos \theta} \\
\cos (\angle R)=\frac{a \cos \theta-c}{a-c \cos \theta}, & \sin (\angle R)=\frac{b \sin \theta}{a-c \cos \theta}
\end{array}
$$

